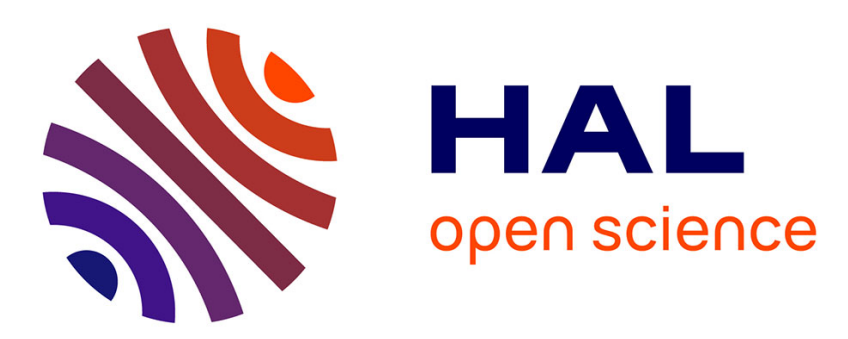

\title{
A linear programming approach to increasing the weight of all minimum spanning trees
}

Mourad Baïou, Fancisco Barahona

\section{To cite this version:}

Mourad Baïou, Fancisco Barahona. A linear programming approach to increasing the weight of all minimum spanning trees. 2005. hal-00242975

\section{HAL Id: hal-00242975 \\ https://hal.science/hal-00242975}

Preprint submitted on 6 Feb 2008

HAL is a multi-disciplinary open access archive for the deposit and dissemination of scientific research documents, whether they are published or not. The documents may come from teaching and research institutions in France or abroad, or from public or private research centers.
L'archive ouverte pluridisciplinaire HAL, est destinée au dépôt et à la diffusion de documents scientifiques de niveau recherche, publiés ou non, émanant des établissements d'enseignement et de recherche français ou étrangers, des laboratoires publics ou privés. 


\title{
ECOLE POLYTECHNIQUE
}

CENTRE NATIONAL DE LA RECHERCHE SCIENTIFIQUE

\section{A linear programming approach to increasing the weight of all minimum spanning trees}

\author{
Mourad Baïou \\ Francisco Barahona
}

May 2005

Cahier $n^{\circ}$ 2005-012

\section{LABORATOIRE D'ECONOMETRIE}

1rue Descartes F-75005 Paris

(33) 155558215

http://ceco.polytechnique.fr/

mailto:labecox@poly.polytechnique.fr 


\title{
A linear programming approach to increasing the weight of all minimum spanning trees
}

\author{
Mourad Baïou ${ }^{1}$ \\ Francis Barahona
}

May 2005

Cahier $n^{\circ}$ 2005-012

Résumé: Dans cet article nous étudions le problème qui consiste à augmenter au moindre coût le poids de tous les arbres couvrants de poids minimum. Nous considérons le cas où le coût d'augmenter le poids d'une arête du graphe est une fonction linéaire par morceaux, convexe et croissante. Nous formulons ce problème par un programme linéaire et nous donnons un algorithme polynomial pour sa résolution et la résolution du son problème dual.

Abstract: Given a graph where increasing the weight of an edge has a nondecreasing convex piecewise linear cost, we study the problem of finding a minimum cost increase of the weights so that the value of all minimum spanning trees is equal to some target value. We formulate this as a combinatorial linear program and give an algorithm.

Mots clés : $\quad$ Arbres couvrants de poids minimum, sécurisation des réseaux.

Key Words : Minimum weight spanning trees, packing spanning trees, network reinforcement, strength problem.y

Classification AMS:

\footnotetext{
${ }^{1}$ Laboratoire d’Econométrie, CNRS et Ecole polytechnique.
} 


\title{
A LINEAR PROGRAMMING APPROACH TO INCREASING THE WEIGHT OF ALL MINIMUM SPANNING TREES
}

\author{
MOURAD BAÏOU AND FRANCISCO BARAHONA
}

\begin{abstract}
Given a graph where increasing the weight of an edge has a nondecreasing convex piecewise linear cost, we study the problem of finding a minimum cost increase of the weights so that the value of all minimum spanning trees is equal to some target value. We formulate this as a combinatorial linear program and give an algorithm.
\end{abstract}

Keywords: Minimum weight spanning trees, packing spanning trees, network reinforcement, strength problem.

\section{INTRODUCTION}

We deal with a graph $G=(V, E)$ where each edge $e \in E$ has an original weight $w_{e}^{0}$ and we can assign to $e$ a new weight $w_{e} \geq w_{e}^{0}$. The cost of giving the weight $w_{e}$ is $c_{e}\left(w_{e}\right)$. The function $c_{e}(\cdot)$ is nondecreasing, convex, piecewise linear and $c_{e}\left(w_{e}^{0}\right)=0$, see Figure 1. We study the following problem: Given a value $\lambda \geq 0$ find a minimum cost set of weights so that the weight of a minimum spanning tree is $\lambda$. We formulate this as a parametric linear program and study its properties.

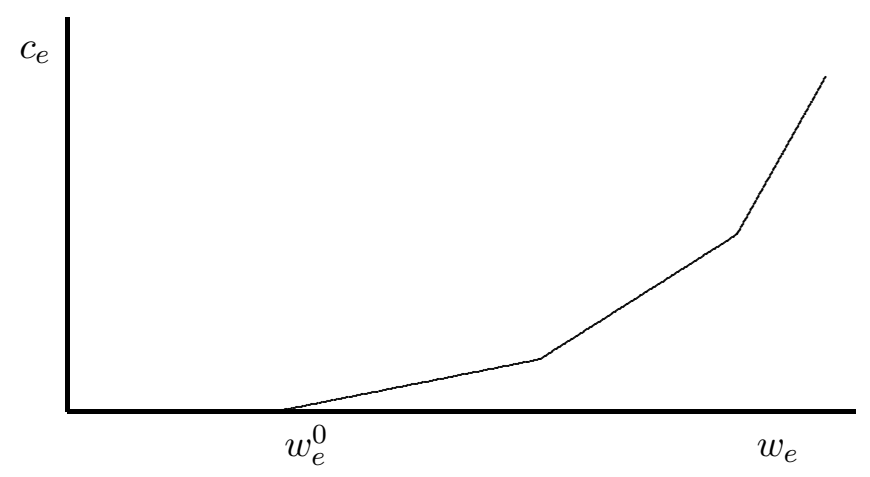

Figure 1. Cost of increasing the weight of an edge.

Frederickson and Solis-Oba [6] studied the case when $c_{e}(\cdot)$ is linear and nondecreasing, so our algorithm is a slight generalization of the one given by them. We study a linear programming formulation of this problem and show how to construct a primal and a dual solution. We also show the relation 
between this and other combinatorial problems like network reinforcement and packing spanning trees.

This paper is organized as follows. In Section 2 we give the linear programming formulation. In Section 3 we deal with related combinatorial problems. In Section 4 we describe the network reinforcement problem. In Section 5 we give the algorithm that builds a primal and a dual solution.

The rest of this section is devoted to some definitions and notation. For a family of disjoint node sets $\left\{S_{1}, \ldots, S_{p}\right\}$ we denote by $\delta\left(S_{1}, \ldots, S_{p}\right)$ the set of edges with both endpoints in different sets of this family. Sometimes we shall use the notation $\delta_{G}\left(S_{1}, \ldots, S_{p}\right)$ to express the fact that this edge set corresponds to edges in $G$. We are going to write $\delta\left(v_{1}, \ldots, v_{l}\right)$ instead of $\delta\left(\left\{v_{1}\right\}, \ldots,\left\{v_{l}\right\}\right)$. For a vector $x \in \mathbb{R}^{E}$ and a subset $A \subseteq E$, we denote $\sum_{a \in A} x(a)$ by $x(A)$. If $F \subset E$ then $G^{\prime}=(V, F)$ is called a spanning subgraph. If $W \subset V$, and $E(W)$ is the set of edges with both endnodes in $W$, then $G(W)=(W, E(W))$ is called the subgraph induced by $W$. We denote by $n$ the number of nodes of $G$, and by $m$ the number of edges of $G$. We abbreviate "minimum weight spanning tree" by MWST.

\section{INCREASING THE WEIGHT OF MWSTS: A LINEAR PROGRAM}

For every edge $e$ we have a convex nondecreasing piecewise linear cost function of the weight $w_{e}$. This is easy to model using linear programming as follows. Assume that for every edge $e$ there are $m_{e}$ possible slopes $d_{e}^{1}, \ldots, d_{e}^{m_{e}}$ of $c_{e}(\cdot)$. For the value $\bar{w}$ the $\operatorname{cost} c_{e}(\bar{w})$ can be obtained as the optimal value of

$$
\begin{aligned}
& \min \sum_{k} d_{e}^{k} x_{e}^{k} \\
& \sum_{k} x_{e}^{k}+w_{e}^{0}=\bar{w} \\
& 0 \leq x_{e}^{k} \leq u_{e}^{k}, \quad 1 \leq k \leq m_{e} .
\end{aligned}
$$

We assume that $d_{e}^{k}<d_{e}^{k+1}$, for $k=1, \ldots, m_{e}-1$. The solution $\bar{x}$ of this linear program is as follows:

there is an index $k_{e} \geq 1$ such that

$$
\begin{aligned}
& \bar{x}_{e}^{k}=u_{e}^{k}, \text { for } 1 \leq k \leq k_{e}-1, \\
& u_{e}^{k_{e}}>\bar{x}_{e}^{k_{e}}=\bar{w}-w_{e}^{0}-\sum_{1 \leq k \leq k_{e}-1} u_{e}^{k} \geq 0,
\end{aligned}
$$$$
\bar{x}_{e}^{k}=0, \text { for } k_{e}+1 \leq k \leq m_{e} .
$$ 
Thus our problem can be modeled as

$$
\begin{aligned}
& \min d x \\
& \sum_{e \in T} w_{e} \geq \lambda, \text { for each tree } T \\
& w_{e}=w_{e}^{0}+\sum_{k=1}^{m_{e}} x_{e}^{k}, \text { for each edge } e \\
& 0 \leq x \leq u
\end{aligned}
$$

This is equivalent to

$$
\begin{aligned}
& \min d x \\
& w^{0}(T)+\sum_{e \in T} \sum_{k=1}^{m_{e}} x_{e}^{k} \geq \lambda, \text { for each tree } T \\
& 0 \leq x \leq u
\end{aligned}
$$

This paper is devoted to the study of the linear program (12)-(14) and its connections with other problems from polyhedral combinatorics.

The dual problem is

$$
\begin{aligned}
& \max \sum\left(\lambda-w^{0}(T)\right) y_{T}-\sum \alpha_{e}^{k} u_{e}^{k} \\
& \sum_{T: e \in T} y_{T} \leq d_{e}^{k}+\alpha_{e}^{k}, \quad 1 \leq k \leq m_{e}, e \in E \\
& y, \alpha \geq 0 .
\end{aligned}
$$

If $\bar{x}$ is an optimal solution of (12)-(14), it satisfies (5)-(7). So if $(\bar{y}, \bar{\alpha})$ is an optimal solution of (15)-(17), the complementary slackness conditions are as follows: for each edge $e$ let $k_{e}$ be defined as in (4), then

$$
\begin{aligned}
& \sum_{T: e \in T} \bar{y}_{T} \geq d_{e}^{k}, \text { for } 1 \leq k \leq k_{e}-1, \\
& \text { if } 0<\bar{x}_{e}^{k_{e}}<u_{e}^{k_{e}} \text { then } \sum_{T: e \in T} \bar{y}_{T}=d_{e}^{k_{e}}, \\
& \sum_{T: e \in T} \bar{y}_{T} \leq d_{e}^{k}, \text { for } k_{e} \leq k \leq m_{e}, \\
& \text { if } \bar{y}_{T}>0 \text { then } \sum_{e \in T} \sum_{k} \bar{x}_{e}^{k}=\lambda-w^{0}(T) .
\end{aligned}
$$

For a weight $w_{e}$ let $c_{e}^{-}\left(w_{e}\right)$ and $c_{e}^{+}\left(w_{e}\right)$ be the left-hand and right-hand derivatives of $c_{e}$ at the value $w_{e}$. Notice that $c_{e}^{-}\left(w_{e}\right) \leq c_{e}^{+}\left(w_{e}\right)$ and the strict inequality holds at the breakpoints. With this notation conditions (18)-(20) can be written as

$$
c_{e}^{-}\left(w_{e}\right) \leq \sum_{T: e \in T} \bar{y}_{T} \leq c_{e}^{+}\left(w_{e}\right) .
$$




\section{Related COMbinatorial PROBlems}

3.1. Kruskal's algorithm for MWSTs. We describe Kruskal's algorithm [11] for MWSTs, this will be needed in the following sections. Assume that we have a graph $G=(V, E)$ with edge weights $w_{e}$ for $e \in E$, and the weights take values $\omega_{1}<\omega_{2}<\ldots<\omega_{r}$. Let

$$
F_{i}=\left\{e \in E \mid w_{e}=\omega_{i}\right\} .
$$

We can describe Kruskal's algorithm for MWSTs as follows. Let $G_{1}, \cdots, G_{p}$ be the subgraphs given by the connected components of the spanning subgraph defined by $F_{1}$, find a spanning tree in each graph $G_{i}$ and shrink it to a single node. Repeat the same with $F_{2}$ and so on. All MWSTs can be obtained in this way. We illustrate this in Figure 2; the numbers close to the edges are their weights, we also show the nested family of node sets that are being shrunk.

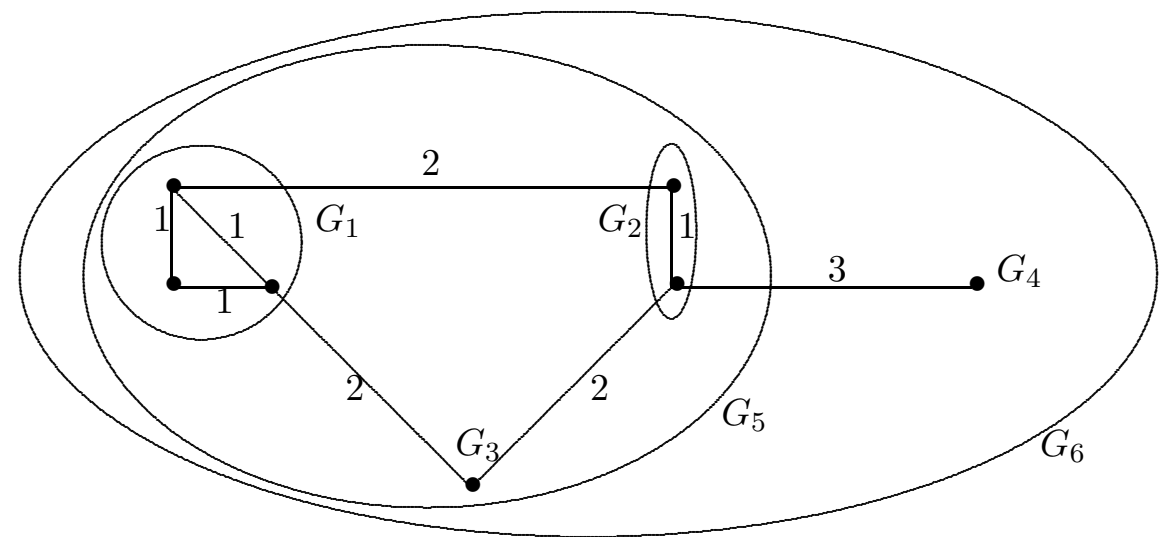

FIGURE 2. The subgraphs being shrunk in Kruskal's algorithm.

We denote by $\left\{G_{i}\right\}$ the family of subgraphs produced by this algorithm.

3.2. Packing spanning trees. Given a graph $G=(V, E)$ with nonnegative edge costs $d_{e}$ for $e \in E$, we consider the linear program

$$
\begin{aligned}
& \min d x \\
& x(T) \geq 1, \text { for all spanning trees } T \\
& x \geq 0 .
\end{aligned}
$$

Its dual is

$$
\begin{aligned}
& \max \sum y_{T} \\
& \sum_{\substack{T: e \in T \\
y \geq 0}} y_{T} \leq d_{e}, \text { for all } e \\
&
\end{aligned}
$$


This last problem can be seen as a packing of spanning trees with capacities $d$. The value of the dual objective function is the value of the packing.

Let $\left\{S_{1}, \cdots, S_{p}\right\}$ be a partition of $V$, let $\bar{x}$ be a vector defined as

$$
\bar{x}_{e}= \begin{cases}\frac{1}{p-1} & \text { if } e \in \delta\left(S_{1}, \cdots, S_{p}\right) \\ 0 & \text { otherwise. }\end{cases}
$$

It follows from the results of [13] and [12] that the extreme points of the polyhedron defined by (24)-(25) are as in (29). Thus solving the linear program (23)-(25) is equivalent to

$$
\min \frac{d\left(\delta\left(S_{1}, \cdots, S_{p}\right)\right)}{p-1}
$$

where the minimum is taken over all partitions $\left\{S_{1}, \cdots, S_{p}\right\}$ of $V$. This was called the strength problem in [5].

It follows from linear programming duality that the value of the minimum in (30) is equal to the value of a maximum packing of spanning trees with capacities $d$. Also if $\bar{y}$ is a maximum packing and $\left\{S_{1}, \cdots, S_{p}\right\}$ is a solution of (30), then

$$
\sum_{T: e \in T} \bar{y}_{T}=d_{e}
$$

for each edge $e \in \delta\left(S_{1}, \cdots, S_{p}\right)$.

Algorithms for the strength problem have been given in [5], [9], [7] and [4]. The last two references give $O\left(n^{4}\right)$ algorithms. For the dual problem (26)-(28) $O\left(n^{5}\right)$ algorithms have been given in [2] and [8].

The following observation will be used later. Let $\tilde{y}$ be a vector that satisfies (27)-(28). Let $k=\sum_{T} \tilde{y}_{T}$, and

$$
d_{e}^{\prime}=\sum_{T: e \in T} \tilde{y}_{T}
$$

Then

$$
d^{\prime}(E)=k(n-1)
$$

and

$$
d^{\prime}\left(\delta\left(S_{1}, \cdots, S_{p}\right)\right) \geq k(p-1)
$$

for any partition $\left\{S_{1}, \cdots, S_{p}\right\}$ of $V$.

3.3. A simple case. Here we discuss a simpler version of problem (12)(14). Later we shall see that the original problem reduces to a sequence of problems of the simpler type.

Assume that every edge has the same original weight $w^{0}$ and that $x_{e}$ is the amount by which the weight can be increased with a per unit cost $d_{e}$. 
Then (12)-(14) can be written as

$$
\begin{aligned}
& \sum_{e \in T}^{\min } d x \\
& x \geq 0
\end{aligned}
$$

or

$$
\begin{aligned}
& \sum_{e \in T}^{\min } d x \\
& x \geq 0
\end{aligned}
$$

that is equivalent to $(23)-(25)$ when $\lambda>(n-1) w^{0}$.

3.4. The attack problem. Given a set of nonnegative weights $u_{e}$ for all $e \in E$, and a nonnegative number $k$ consider

$$
\min u\left(\delta\left(S_{1}, \ldots, S_{p}\right)\right)-k(p-1),
$$

where the minimization is done over all partitions $\left\{S_{1}, \ldots, S_{p}\right\}$ of $V$. This has been called the attack problem in [5]. An $O\left(n^{5}\right)$ algorithm was given in [5] and later an $O\left(n^{4}\right)$ was given in [1]. We show here some characteristics of the solutions of the attack problem (36), these appear in [3].

Lemma 1. Let $\Phi=\left\{S_{1}, \ldots, S_{p}\right\}$ be a solution of (36), and let $\left\{T_{1}, \ldots, T_{q}\right\}$ be a partition of $S_{i}$, for some $i, 1 \leq i \leq p$. Then

$$
u\left(\delta\left(T_{1}, \ldots, T_{q}\right)\right)-k(q-1) \geq 0 .
$$

Proof. If $u\left(\delta\left(T_{1}, \ldots, T_{q}\right)\right)-k(q-1)<0$ one could improve the solution of (36) by removing $S_{i}$ from $\Phi$ and adding $\left\{T_{1}, \ldots, T_{q}\right\}$.

Lemma 2. Let $\Phi=\left\{S_{1}, \ldots, S_{p}\right\}$ be a solution of (36), and let $\left\{S_{i_{1}}, \ldots, S_{i_{l}}\right\}$ be a sub-family of $\Phi$. Then

$$
u\left(\delta\left(S_{i_{1}}, \ldots, S_{i_{l}}\right)\right)-k(l-1) \leq 0 .
$$

Proof. If $u\left(\delta\left(S_{i_{1}}, \ldots, S_{i_{l}}\right)\right)-k(l-1)>0$, one could improve the solution of (36) by removing $\left\{S_{i_{1}}, \ldots, S_{i_{l}}\right\}$ from $\Phi$ and adding their union.

Lemma 3. If $u(E)=k(n-1)$ and $u\left(\delta\left(S_{1}, \cdots, S_{p}\right)\right) \geq k(p-1)$ for every partition $\left\{S_{1}, \cdots, S_{p}\right\}$ of $V$ then for $k^{\prime} \geq k$ the solution of

$$
\min u\left(\delta\left(S_{1}, \cdots, S_{p}\right)\right)-k^{\prime}(p-1)
$$

is the partition of all singletons. The same is true if some edges are deleted before solving (37).

Proof. Since a solution of (36) is the partition of all singletons, it follows from Lemma 2 that

$$
u\left(\delta\left(v_{1}, \cdots, v_{l}\right)\right)-k(l-1) \leq 0,
$$


for any set of nodes $\left\{v_{1}, \cdots, v_{l}\right\}$. Therefore

$$
u\left(\delta\left(v_{1}, \cdots, v_{l}\right)\right)-k^{\prime}(l-1) \leq 0 .
$$

Thus when solving (37), for any partition $\left\{S_{1}, \cdots, S_{p}\right\}$, if $\left|S_{j}\right|>1$ it follows from Lemma 1 that one can obtain a partition that is not worse by replacing $S_{j}$ by all singletons included in $S_{j}$. The same is true if some edges are deleted before solving (37).

Lemma 4. Let $\Phi=\left\{S_{1}, \ldots, S_{p}\right\}$ be a solution of (36) in $G$. Let $G^{\prime}$ be the graph obtained by adding one new edge e to $G$. If there is an index $i$ such that $e \subseteq S_{i}$ then $\Phi$ is a solution of (36) in $G^{\prime}$, otherwise a solution of (36) in $G^{\prime}$ is of the form

$$
\Phi^{\prime}=\left(\Phi \backslash\left\{S_{i}: i \in I\right\}\right) \cup\left\{U=\cup_{i \in I} S_{i}\right\},
$$

for some index set $I \subseteq\{1, \ldots, p\}$, and $e \in \delta\left(S_{i_{1}}, S_{i_{2}}\right)$, with $\left\{i_{1}, i_{2}\right\} \subseteq I$. The set I could be empty, in which case $\Phi^{\prime}=\Phi$. See Figure 3.

Proof. Let $\left\{T_{1}, \ldots, T_{q}\right\}$ be a solution of (36) in $G^{\prime}$. Assume that there is a set $S_{i}$ such that $S_{i} \subseteq \cup_{l=1}^{l=r} T_{j_{l}}, r \geq 2$, and $S_{i} \cap T_{j_{l}} \neq \emptyset$ for $1 \leq l \leq r$. Lemma 1 implies that

$$
u\left(\delta_{G}\left(T_{j_{1}} \cap S_{i}, \ldots, T_{j_{r}} \cap S_{i}\right)\right)-k(r-1) \geq 0,
$$

and $u\left(\delta_{G^{\prime}}\left(T_{j_{1}}, \ldots, T_{j_{r}}\right)\right)-k(r-1) \geq 0$. Therefore $\left\{T_{j_{1}}, \ldots, T_{j_{r}}\right\}$ can be replaced by their union. So we can assume that for all $i$ there is an index $j(i)$ such that $S_{i} \subseteq T_{j(i)}$.

Now suppose that for some index $j, T_{j}=\cup_{q=1}^{q=l} S_{i_{q}}, l>1$. If $e \notin$ $\delta_{G^{\prime}}\left(S_{i_{1}}, \ldots, S_{i_{l}}\right)$, from Lemma 2 we have that

$$
u\left(\delta_{G^{\prime}}\left(S_{i_{1}}, \ldots, S_{i_{l}}\right)\right)-k(l-1) \leq 0,
$$

and we could replace $T_{j}$ by $\left\{S_{i_{1}}, \ldots, S_{i_{l}}\right\}$. If $e \in \delta_{G^{\prime}}\left(S_{i_{1}}, \ldots, S_{i_{l}}\right)$, only then we could have

$$
u\left(\delta_{G^{\prime}}\left(S_{i_{1}}, \ldots, S_{i_{l}}\right)\right)-k(l-1)>0,
$$

and we should keep $T_{j} \in \Phi^{\prime}$.

\section{NETWORK REINFORCEMENT}

The network reinforcement problem is defined in a graph $G=(V, E)$ with edge costs $d$, edge capacities $u$ and a nonnegative number $k$ called the target. It consists of finding a subgraph of minimum weight that contains $k$ disjoint spanning trees. Multiple copies of each edge can be used; for each edge $e$ the number of copies to be used is bounded by $u_{e}$. This problem has been studied in [5], [7] and [3]. The last two references give $O\left(n^{4}\right)$ algorithms. Below we describe the algorithm of [3]. This will be needed in the following section. 


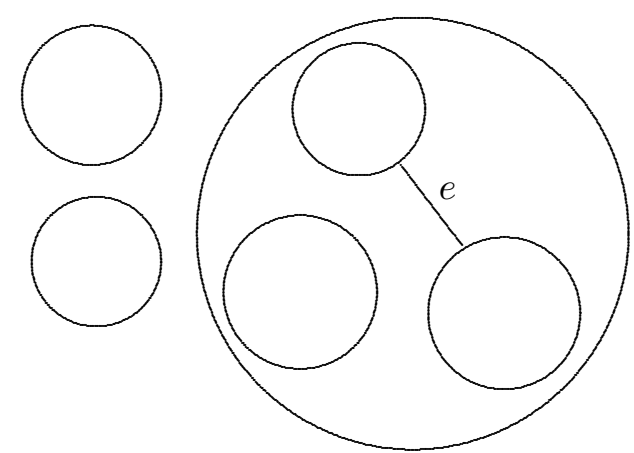

Figure 3 . The family $\Phi^{\prime}$ is obtained by combining some sets in $\Phi$.

We solve the linear program

$$
\begin{aligned}
& \operatorname{minimize} d x \\
& \text { subject to } \\
& x\left(\delta\left(S_{1}, \ldots, S_{p}\right)\right) \geq k(p-1), \\
& \text { for all partitions } \Phi=\left\{S_{1}, \ldots, S_{p}\right\} \text { of } V, \\
& 0 \leq x_{e} \leq u_{e} .
\end{aligned}
$$

Instead of using inequalities (40), we use the equivalent extended formulation proposed in [10] as follows. Associate variables $y$ with the nodes and variables $x$ with the edges of the graph, choose an arbitrary node $r$, and solve the linear program below.

$$
\begin{aligned}
& \min \sum d x \\
& x(\delta(S))+y(S) \geq\left\{\begin{array}{ll}
2 k & \text { if } r \notin S, \\
0 & \text { if } r \in S,
\end{array} \text { for all } S \subseteq V,\right. \\
& y(V)=0, \\
& -x \geq-u, \\
& x \geq 0 .
\end{aligned}
$$

Its dual is

$$
\begin{aligned}
& \max \sum_{\{S: r \notin S\}} 2 k w_{S}-\sum u_{e} \beta_{e} \\
& \sum_{\{S: e \in \delta(S)\}} w_{S} \leq d_{e}+\beta_{e} \quad \text { for all } e \in E, \\
& \sum_{\{S: v \in S\}} w_{S}=\mu \quad \text { for all } v, \\
& w \geq 0, \quad \beta \geq 0, \quad \mu \text { unrestricted. }
\end{aligned}
$$


A dual algorithm will be used, constraints (48), (49) and (50) will always be satisfied and we are going to maximize (47). For the primal problem, constraints (43), (45), and (46) will always be satisfied, and (44) will be satisfied at the end. Complementary slackness will be kept at all stages. We start with an informal description of the algorithm.

At the beginning we set to zero all dual variables. We are going to choose a partition $\left\{S_{1}, \ldots, S_{p}\right\}$ of $V$ and increase by $\epsilon$ the value of the variables $\left\{w_{S_{i}}\right\}$. This will ensure that constraint (49) is satisfied. We have to ensure that constraints (48) are satisfied for all $e \in \delta\left(S_{1}, \ldots, S_{p}\right)$. We say that an edge $e$ is tight if its constraint (48) is satisfied as equation. For a tight edge $e \in \delta\left(S_{1}, \ldots, S_{p}\right)$ we have to increase the value of $\beta_{e}$ by $2 \epsilon$. Let $H$ be the subgraph defined by the tight edges. The objective function changes by

$$
\epsilon\left(2 k(p-1)-2 u\left(\delta_{H}\left(S_{1}, \ldots, S_{p}\right)\right)\right) .
$$

So one should find a partition $\left\{S_{1}, \ldots, S_{p}\right\}$ of $V$ such that

$$
k(p-1)-u\left(\delta_{H}\left(S_{1}, \ldots, S_{p}\right)\right)>0 .
$$

Thus we solve

$$
\text { minimize } u\left(\delta_{H}\left(S_{1}, \ldots, S_{p}\right)\right)-k(p-1),
$$

among all partitions $\left\{S_{1}, \ldots, S_{p}\right\}$ of $V$. This is problem (36) Let $\Phi=$ $\left\{S_{1}, \ldots, S_{p}\right\}$ be the solution obtained. Let $(\bar{w}, \bar{\beta}, \bar{\mu})$ be the current dual solution. If the minimum in (51) is negative we use the largest value of $\epsilon$ so that a new edge becomes tight. This is

$$
\bar{\epsilon}=\frac{1}{2} \min \left\{\bar{d}_{e}=d_{e}-\sum_{\{S: e \in \delta(S)\}} \bar{w}_{S} \mid e \in \delta_{G}\left(S_{1}, \ldots, S_{p}\right) \backslash \delta_{H}\left(S_{1}, \ldots, S_{p}\right)\right\} .
$$

If this minimum is taken over the empty set we say that $\bar{\epsilon}=\infty$. In this case the dual problem is unbounded and the primal problem is infeasible. Notice that $\bar{\beta}_{e}=0$ if $e$ is not tight, and when an edge becomes tight it remains tight.

Now assume that an edge $e=\{v, q\}$ gives the minimum in (52). If there is more than one edge achieving the minimum in (52) we pick arbitrarily one. Let $\Phi^{\prime}$ be the solution of (51) after adding $e$ to $H$. If $\Phi^{\prime}=\Phi$ then $\beta_{e}$ could increase and $x_{e}$ should take the value $u_{e}$, to satisfy complementary slackness; we call this Case 1. Otherwise according to Lemma 4 we have that

$$
\Phi^{\prime}=\left(\Phi \backslash\left\{S_{i}: i \in I\right\}\right) \cup\left\{U=\cup_{i \in I} S_{i}\right\},
$$

for some index set $I \subseteq\{1, \ldots, p\}$, and $e \in \delta\left(S_{i_{1}}, S_{i_{2}}\right)$, with $\left\{i_{1}, i_{2}\right\} \subseteq I$. If so $\beta_{e}$ remains equal to zero and $x_{e}$ can take a value less than $u_{e}$, this is called Case 2. The algorithm stops when the minimum in (51) is zero.

Now we have to describe how to produce a primal solution. At any stage we are going to have a vector $(\bar{y}, \bar{x})$ satisfying (43), (45), and (46). Equation (44) will be satisfied only at the end. 
Complementary slackness will be maintained throughout the algorithm. For (43), we need that for each set $S$ with $\bar{w}_{S}>0$ the corresponding inequality holds as equation. Also we can have $\bar{x}_{e}>0$ only if $e$ is tight, and if $\bar{\beta}_{e}>0$ we should have $\bar{x}_{e}=u_{e}$.

Initially we set $\bar{x}=0, \bar{y}_{u}=2 k$ if $u \neq r$ and $\bar{y}_{r}=0$. We have to discuss the update of $(\bar{y}, \bar{x})$ in cases 1 and 2 above.

In Case 1 , we set $\bar{x}_{e}=u_{e}$ and update $\bar{y}$ as $\bar{y}_{v} \leftarrow \bar{y}_{v}-u_{e}, \bar{y}_{q} \leftarrow \bar{y}_{q}-u_{e}$. Thus for any set $S$ such that $e \in \delta(S)$, if its corresponding inequality (43) was tight, it will remain tight.

In Case 2, we have that

$$
\Phi^{\prime}=\left(\Phi \backslash\left\{S_{i}: i \in I\right\}\right) \cup\left\{U=\cup_{i \in I} S_{i}\right\},
$$

for some index set $I \subseteq\{1, \ldots, p\}$, and $e \in \delta\left(S_{i_{1}}, S_{i_{2}}\right)$, with $\left\{i_{1}, i_{2}\right\} \subseteq I$. Let

$$
\lambda= \begin{cases}\bar{x}(\delta(U))+\bar{y}(U)-2 k & \text { if } r \notin U, \\ \bar{x}(\delta(U))+\bar{y}(U) & \text { if } r \in U .\end{cases}
$$

We update $(\bar{y}, \bar{x})$ as $\bar{y}_{v} \leftarrow \bar{y}_{v}-\lambda / 2, \bar{y}_{q} \leftarrow \bar{y}_{q}-\lambda / 2$, and $\bar{x}_{e}=\lambda / 2$. Thus the set $U$ becomes tight. The new vector satisfies (43), this is shown in [3].

So at every iteration a new edge becomes tight. In some cases some sets in the family $\Phi$ are combined into one. When this family consists of only the set $V$ then we have that $\bar{y}(V)=0$ and we have a primal feasible solution that together with the dual solution satisfy complementary slackness. The formal description of the algorithm is below.

\section{Network Reinforcement}

- Step 0. Start with $\bar{w}=0, \bar{\beta}=0, \bar{\mu}=0, \bar{y}_{v}=2 k$ if $v \neq r, \bar{y}_{r}=0$, $\bar{x}=0, \bar{d}_{e}=d_{e}$ for all $e \in E, \Phi$ consisting of all singletons, and $H=(V, \emptyset)$.

- Step 1. Compute $\bar{\epsilon}$ as in (52). If $\bar{\epsilon}=\infty$ stop, the problem is infeasible.

Otherwise update $\bar{w}_{S_{i}} \leftarrow \bar{w}_{S_{i}}+\bar{\epsilon}$ for $S_{i} \in \Phi$, $\bar{\beta}_{e} \leftarrow \bar{\beta}_{e}+2 \bar{\epsilon}$ for all $e \in \delta_{H}\left(S_{1}, \ldots, S_{p}\right)$, $\bar{\mu} \leftarrow \bar{\mu}+\bar{\epsilon}$, $\bar{d}_{e} \leftarrow \bar{d}_{e}-2 \bar{\epsilon}$ for all $e \in \delta_{G}\left(S_{1}, \ldots, S_{p}\right) \backslash \delta_{H}\left(S_{1}, \ldots, S_{p}\right)$.

- Step 2. Let $e$ be an edge giving the minimum in (52), add $e$ to $H$. Solve problem (51) in $H$ to obtain a partition $\Phi^{\prime}$.

- Step 3. If $\Phi=\Phi^{\prime}$ update $(\bar{y}, \bar{x})$ as in Case 1 . Otherwise update as in Case 2. If $\Phi^{\prime}=\{V\}$ stop, the equation $\bar{y}(V)=0$ is satisfied. Otherwise set $\Phi \leftarrow \Phi^{\prime}$ and go to Step 1 .

Since at every iteration a new edge becomes tight, this algorithm takes at most $|E|$ iterations.

\section{Producing a primal and a dual Vector}

5.1. General Procedure. We are going to solve (8)-(11) or (12)-(14) as a parametric linear program with parameter $\lambda$. First we set $w=w^{0}, x=0$, $y=0$, and $\lambda$ equal to the value of a MWST with weights $w^{0}$. 
Then we assume that for $\lambda=\lambda^{1} \geq 0$ we have an optimal primal solution $w^{1}$, and an optimal dual solution $y^{1}$. We have that if $y_{T}^{1}>0$ then

$$
\sum_{e \in T} w_{e}^{1}=\lambda^{1}
$$

and

$$
c_{e}^{-}\left(w_{e}^{1}\right) \leq \sum_{T: e \in T} y_{T}^{1} \leq c_{e}^{+}\left(w_{e}^{1}\right),
$$

for each edge $e$.

Let $\left\{G_{i}\right\}$ be the family of graphs produced by Kruskal's algorithm with weights $w^{1}$. In order to increase $\lambda$ by a small amount we have to increase for some $G_{i}$ the weight of every MWST. Since all weights in $G_{i}$ are the same, our problem reduces to (33)-(35) or (23)-(25). So we have to solve (30), where the cost of each edge $e$ is $c^{+}\left(w_{e}^{1}\right)$.

Let $G_{i}=\left(V_{i}, E_{i}\right)$. For a partition $P=\left\{S_{1}, \cdots, S_{p}\right\}$ of $V_{i}$ and a vector $w$ we define

$$
c^{+}\left(\delta\left(S_{1}, \cdots, S_{p}\right), w\right)=\sum_{e \in \delta\left(S_{1}, \cdots, S_{p}\right)} c_{e}^{+}\left(w_{e}\right) .
$$

For each graph $G_{i}$ we compute $\sigma_{i}$ as the value of the solution of

$$
\min c^{+}\left(\delta\left(T_{1}^{i}, \cdots, T_{p}^{i}\right), w^{1}\right) /(p-1),
$$

among all partitions $\left\{T_{1}^{i}, \cdots, T_{p}^{i}\right\}$ of $V_{i}$.

Let $j=\operatorname{argmin}\left\{\sigma_{i}\right\}$, and $\tau=\sigma_{j}$. Let $\left\{T_{1}^{j}, \cdots, T_{p}^{j}\right\}$ be a partition of $V_{j}$ that is a solution of (55). Then $\lambda$ is increased by a small value $\epsilon$ and the weights of all edges in $\delta_{G_{j}}\left(T_{1}^{j}, \cdots, T_{p}^{j}\right)$ is increased by $\epsilon /(p-1)$.

Now we have to produce a new dual solution that proves the optimality of the new vector $w$. For that we are going to produce a packing of spanning trees of value $\tau$ in each graph $G_{i}$ and then combine them into a dual vector for the entire graph. First for each graph $G_{i}$ we are going to compute pseudo $\operatorname{costs} c^{\prime}$ that will be used to find the right packing of spanning trees. For that we solve the network reinforcement problem with target value $\tau$. For each edge $e$ we define

$$
c_{e}^{0}=\sum_{T: e \in T} y_{T}^{1}
$$

as its capacity and a cost equal to 1 . If $c^{+}\left(w_{e}^{1}\right)>c_{e}^{0}$ we add a parallel edge with capacity $c^{+}\left(w_{e}^{1}\right)-c_{e}^{0}$ and cost $M$, a big number. This problem is feasible because when all capacities are used we obtain a graph that admits a packing of spanning trees of value greater or equal to $\tau$. We need the following lemma.

Lemma 5. Let $c^{\prime}$ be the solution of the network reinforcement problem then $c^{+}\left(w_{e}^{1}\right) \geq c_{e}^{\prime} \geq c_{e}^{0}$ for all $e$. 
Proof. The proof is based on the algorithm of Section 4. It starts with the partition $\Phi$ consisting of all singletons. Then the dual variables associated to all sets in $\Phi$ take the value $1 / 2$. Then one edge $e$ with cost 1 becomes tight and its primal variable is set to its upper bound $c_{e}^{0}$. Now we have to see that the algorithm will continue to produce the same partition $\Phi$ until all edges with cost 1 become tight.

Let $k=\sum_{T} y_{T}^{1}$ and $k^{\prime}=\tau$. We have that $k^{\prime} \geq k$ because $k^{\prime}$ is the value of a maximum packing of spanning trees in $G_{j}$ with capacity $c^{+}\left(w_{e}^{1}\right)$ for each edge $e$, and $y^{1}$ is a packing that satisfies

$$
\sum_{T: e \in T} y_{T}^{1} \leq c_{e}^{+}\left(w_{e}^{1}\right)
$$

for each edge $e$.

Here $G_{i}=\left(V_{i}, E_{i}\right)$ with $V_{i}=\left\{v_{1}^{\prime}, \cdots, v_{p}^{\prime}\right\}$. We have that

$$
c^{0}\left(\delta\left(v_{1}^{\prime}, \ldots, v_{p}^{\prime}\right)\right)=k(p-1)
$$

for the trivial partition, see (31); and

$$
c^{0}\left(\delta\left(S_{1}, \ldots, S_{q}\right)\right) \geq k(q-1)
$$

for any other partition $\left\{S_{1}, \ldots, S_{q}\right\}$ of $V_{i}$, see (32). Lemma 3 implies that the reinforcement algorithm will use the trivial partition until each edge $e$ with cost 1 becomes tight and its primal variable takes the value $c_{e}^{0}$. Since the algorithm never decreases the value of a variable we have $c_{e}^{\prime} \geq c_{e}^{0}$.

The definition of the capacities implies $c^{+}\left(w_{e}^{1}\right) \geq c_{e}^{\prime}$.

When solving the network reinforcement problem we are minimizing so the solution $c^{\prime}$ is minimal, thus there is a packing of spanning trees $y^{i}$ in $G_{i}$ of value $\tau$ and such that

$$
\sum_{T: e \in T} y_{T}^{i}=c_{e}^{\prime}, \text { for all } e \in E_{i} .
$$

Therefore

$$
c_{e}^{-}\left(w_{e}^{1}\right) \leq \sum_{T: e \in T} y_{T}^{1} \leq \sum_{T: e \in T} y_{T}^{i} \leq c_{e}^{+}\left(w_{e}^{1}\right),
$$

for each edge $e \in E_{i}$.

For the graph $G_{j}$ we have that

$$
\sum_{T: e \in T} y_{T}^{j}=c_{e}^{+}\left(w_{e}^{1}\right)
$$

for each edge $e \in \delta_{G_{j}}\left(T_{1}^{j}, \cdots, T_{p}^{j}\right)$. This is because $\tau$ is the value of a maximum packing of spanning trees in $G_{j}$ and all capacities in $\delta_{G_{j}}\left(T_{1}^{j}, \cdots, T_{p}^{j}\right)$ should be used by this packing. 
Then these packings are combined to produce a packing of spanning trees in the original graph as described in the next subsection. This dual solution satisfies the complementary slackness conditions with the new primal solution. This is a proof of optimality.

We can continue increasing $\lambda$ and the weights of the edges in $\delta_{G_{j}}\left(T_{1}^{j}, \cdots, T_{p}^{j}\right)$ until either a breakpoint of the cost function of some edge is found, this is called a Type 1 iteration; or the weights of the edges in $\delta_{G_{j}}\left(T_{1}^{j}, \cdots, T_{p}^{j}\right)$ reach a value equal to the value of the edges in the graph $G_{l}$ containing $G_{j}$, in this later case only the family $\left\{G_{i}\right\}$ changes, this is called a Type 2 iteration. In either case we restart as in the beginning of this section. If none of these cases is found, i. e. there is no limit for increasing $\lambda$, the algorithm stops.

5.2. Combining Dual Vectors. Let $\left\{G_{i}\right\}$ be the family of graphs produced by Kruskal's algorithm, we have to describe how to combine the dual vectors produced for each graph $G_{i}$. This is done as follows.

Let $G_{0}$ be a graph and $G_{1}$ an induced subgraph of $G_{0}$. Let $G_{2}$ be the graph obtained from $G_{0}$ by shrinking $G_{1}$ to a single node. Assume that we have a packing of spanning trees $y^{1}$ of $G_{1}$ and a packing $y^{2}$ of $G_{2}$ both of value $\tau$. We pick $y_{T}^{1}>0$ and $y_{S}^{2}>0$, we set $\mu=\min \left\{y_{T}^{1}, y_{S}^{2}\right\}$ and associate this value to $T \cup S$ that is a tree of the graph $G_{0}$. We subtract $\mu$ from $y_{T}^{1}$ and $y_{S}^{2}$ and continue.

This procedure is applied recursively to the family $\left\{G_{i}\right\}$.

5.3. The algorithm. Now we can give a formal description of the algorithm:

- Step 0. Set $w=w^{0}, x=0, y=0$, and $\lambda$ equal to the value of a MWST with weights $w^{0}$. Set $k_{e}=1$ for each edge $e$.

- Step 1. Let $\omega_{1}<\omega_{2}<\ldots<\omega_{r}$ be the different values of the weights $w$. We set $\omega_{r+1}=\infty$. Let $\left\{G_{i}\right\}$ be the family of graphs produced by Kruskal's algorithm.

For each graph $G_{i}=\left(V_{i}, E_{i}\right)$ compute

$$
\sigma_{i}=\min c^{+}\left(\delta\left(T_{1}^{i}, \cdots, T_{p}^{i}\right), w\right) /(p-1),
$$

among all partitions $\left\{T_{1}^{i}, \cdots, T_{p}^{i}\right\}$ of $V_{i}$.

Let $j=\operatorname{argmin}\left\{\sigma_{i}\right\}$, and $\sigma_{j}=c^{+}\left(\delta\left(T_{1}^{i}, \cdots, T_{p}^{i}\right), w\right) /(p-1)$ for a partition $\left\{T_{1}^{j}, \cdots, T_{p}^{j}\right\}$ of $V_{j}$. Let $\omega_{l}$ be the weight of the edges in $E_{j}, l \leq r$.

- Step 2. Let $\rho_{1}=\min \left\{u_{e}^{k_{e}}-x_{e}^{k_{e}}: e \in \delta_{G_{j}}\left(T_{1}^{j}, \cdots, T_{p}^{j}\right)\right\}$, $\rho=\min \left\{\rho_{1}, \omega_{l+1}-\omega_{l}\right\}$. If $\rho=\infty$, stop.

Otherwise set $x_{e}^{k_{e}} \leftarrow x_{e}^{k_{e}}+\rho, w_{e} \leftarrow w_{e}+\rho$, for all $e \in \delta_{G_{j}}\left(T_{1}^{j}, \cdots, T_{p}^{j}\right)$. If $\rho=\rho_{1}$ go to Step 3, otherwise go to Step 4 .

- Step 3. Set $k_{e} \leftarrow k_{e}+1$ for all $e \in \delta_{G_{j}}\left(T_{1}^{j}, \cdots, T_{p}^{j}\right)$ with $x_{e}^{k_{e}}=u_{e}^{k_{e}}$. Produce a new dual solution as described in Subsection 5.1.

- Step 4. Set $\lambda \leftarrow \lambda+\rho(p-1)$. Go to Step 1. 
5.4. Complexity Analysis. Clearly an upper bound for the number of type 1 iterations is $\sum_{e \in E} m_{e}$. Now we have to derive a bound for the number of type 2 iterations.

Lemma 6. Between any two iterations of type 1 there are at most $(n-1)(m-1)$ iterations of type 2.

Proof. At any stage of the algorithm there are at most $m$ different values for the edge weights. Let $\omega_{1}<\omega_{2} \cdots<\omega_{r}$ be all these values. Let $\rho\left(\omega_{i}\right)$ be the number of edges of weight $\omega_{i}$ in any MWST. At each iteration of type 2 there is an index $i$ such that $\rho\left(\omega_{i}\right)$ decreases and $\rho\left(\omega_{i+1}\right)$ increases. Thus there are at most $(n-1)(m-1)$ consecutive type 2 iterations.

At each iteration one has to solve the strength problem (30) for each graph $G_{i}$. Let $n_{i}$ be the number of nodes of $G_{i}$. Since this family of node sets is nested, we have that $\sum n_{i} \leq 2(n-2)$. So the complexity of this sequence of strength problems is $O\left(n^{4}\right)$.

Finding a packing of spanning trees has a complexity $O\left(n^{5}\right)$, with the same arguments as above we have that the complexity of computing the packings for all graphs $G_{i}$ is $O\left(n^{5}\right)$. We can state the following.

Theorem 1. The complexity of producing the primal solution is $O\left(m n^{5} \sum m_{e}\right)$, and the complexity of producing the dual solution is $O\left(m n^{6} \sum m_{e}\right)$.

\section{ACKNOWLEDGMENTS}

Part of this work was done while the first author was visiting the T. J. Watson Research Center of IBM and while the second author was visiting the Laboratoire d'Econométrie de L'Ecole Polytechnique in Paris. The financial support of both institutions is greatly appreciated.

\section{REFERENCES}

[1] F. Barahona, Separating from the dominant of the spanning tree polytope, Op. Research Letters, 12 (1992), pp. 201-203.

[2] F. Barahona, Packing spanning trees, Math. Oper. Res., 20 (1995), pp. 104-115.

[3] F. Barahona, Network reinforcement, tech. rep., IBM Watson Reserach Center, Yorktown Heights NY 10598. Also in www.optimization-online.org, 2002.

[4] E. Cheng And W. H. Cunningham, A faster algorithm for computing the strength of a network, Inf. Process. Lett., 49 (1994), pp. 209-212.

[5] W. H. Cunningham, Optimal attack and reinforcement of a network, J. of ACM, 32 (1985), pp. 549-561.

[6] G. N. Frederickson And R. Solis-Oba, Increasing the weight of minimum spanning trees, J. Algorithms, 33 (1999), pp. 244-266.

[7] H. N. Gabow, Algorithms for graphic polymatroids and parametric $\bar{s}$-sets, J. Algorithms, 26 (1998), pp. 48-86.

[8] H. N. Gabow And K. S. Manu, Packing algorithms for arborescences (and spanning trees) in capacitated graphs, Math. Programming Ser. B, 82 (1998), pp. 83-109.

[9] D. Gusfield, Computing the strength of a graph, SIAM J. Comput., 20 (1991), pp. 639-654. 
[10] M. Jünger and W. R. Pulleyblank, New primal and dual matching heuristics, Algorithmica, 13 (1995), pp. 357-380.

[11] J. B. KRUSKAL, JR., On the shortest spanning subtree of a graph and the traveling salesman problem, Proc. Amer. Math. Soc., 7 (1956), pp. 48-50.

[12] C. S. J. A. NAsh-Williams, Edge-disjoint spanning trees of finite graphs, J. London Math. Soc., 36 (1961), pp. 445-450.

[13] W. T. TutTe, On the problem of decomposing a graph into $n$ connected factors, J. London Math. Soc., 36 (1961), pp. 221-230.

(M. Baïou) Laboratoire D'Economtrie de l'Ecole Polytechnique, 1 Rue Descartes, 75005 PARIS.

E-mail address, M. Baïou: baiou@custsv.univ-bpclermont.fr

(F. Barahona) IBM T. J. Watson research Center, Yorktown Heights, NY 10589, USA

E-mail address, F. Barahona: barahon@us.ibm.com 